\title{
RF/SS System for Wireless Local Loop
}

\author{
J. N. Matos ${ }^{1,2}$, A. Gameiro ${ }^{1,2}$, R. Silva ${ }^{2}$, J. Figueiredo ${ }^{2}$, Mateo Burgos ${ }^{3}$ and Pablo Dorta ${ }^{3}$ \\ ${ }^{1}$ Dept. Electrónica e Telecomunicações / ${ }^{2}$ Instituto de Telecomunicações \\ Campus Universitário, 3810 Aveiro, Portugal \\ E-mail:jnmatos@ua.pt,amg@ua.pt \\ ${ }^{3}$ Dep. Senales Sistemas y Radiocomunicaciones \\ Universidad Politecnica de Madrid
}

\begin{abstract}
In this communication we deal with the use of direct sequence spread spectrum techniques for radio transmission in the local loop. We present a direct sequence spread-spectrum system that is being developed to transmit signals from ISDN-U [1] interfaces between subscriber premises and the local station. Some attention is devoted to the IF and RF blocks.
\end{abstract}

\section{Introduction}

Traditionally the share of the madio frequency (RF) spectrum has been done by allocating bands, or blocks of spectrum, to various services such as broadcasting, mobile, amateur, satellite among others. Recently, there has been another approach to solve this problem. It is based on the ability of certain modulation methods to share the same band without causing noticeable degree of interference. This method, known as SpreadSpectrum (SS) modulation, is especially useful when used in conjunction with the Code Division Multiple Access (CDMA) technique, which is also called Spread Spectrum Multiple Access (SSMA) [2].

There are other benefits for spreading the spectrum: antijamming, antiinterference, low probability of intercept and high resolution ranging among others [3]. The spread-spectrum techniques have been used for many years in military communications due to the difficult interception. Our days they are used in radio frequency communications although there are a few proposals in base-band communications, as in high bit rate light communications [4]. In radio frequency communications the spread-spectrum techniques have large applications in mobile communications, namely the USA commercial system, IS-95, and will probably be the base for the third generation of mobiles communication systems.

Although the application of spread spectrum in mobile communications has polarized the attention there are many others potential applications.

The use of radio communications in the last loop of the public telecommunication network has risen a significant interest in the last years [5]. Furthermore the use of spread-spectrum techniques in the radio path may mitigate several of the impairments associated with conventional radio transmission techniques such as multipath distortion, eavesdropping, etc.

In this communication we consider the use of direct sequence spread spectrum techniques for radio transmission in the local loop. We present a direct

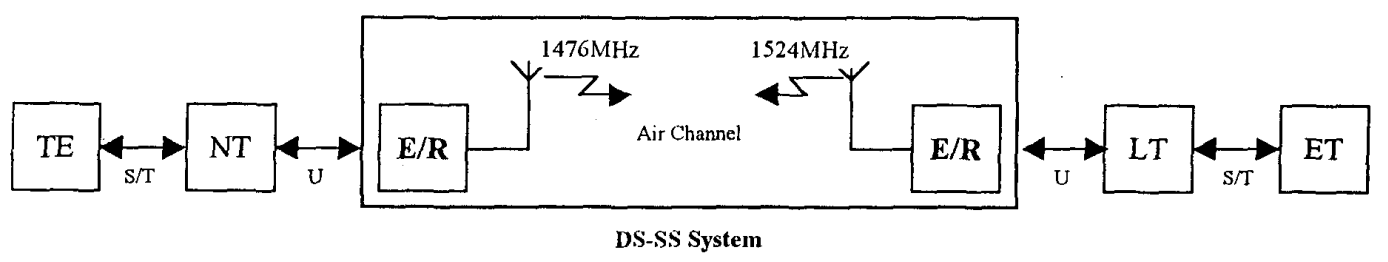

Fig. 1 Insertion of the DS-SS radio system within the ISDN reference configuration 
sequence spread-spectrum system that transmits signals from ISDN-U (Integrated Service Digital Network - U) [1] interfaces between subscriber premises and the local station, Fig. 1.

The system operates in the $1.5 \mathrm{GHz}$ band and is designed to provide a transparent radio path between two $U$ interfaces. Its main specifications are to accommodate up to 10 simultaneous subscribers within $8 \mathrm{Km}$ distance from the local station with no quality degradation compared to a wireline connection. In the communication we present the main design issues and describe the solutions adopted.

The communication is outlined as follows. In the following section the motivations and the justification for the use of spread spectrum radio frequency links in the local loop network are discussed. In section 3 the spread spectrum system that is been developed is shown and in section $4 \mathrm{IF}$ and RF sections are described. Finally in last section the main conclusion of this work are presented.

\section{Local loop SS-RF systems}

Public telecommunications systems are based in a fixed structure from the old twisted copper wired to the high capacity lightwave fiber. This fixed structure corresponds to a large investment that must be profitable.

The use of RF links in local loop may take advantages in the following aspects: the radio frequency technology cost is practically independent of the distance and to set up a RF system it is only necessary to install transmit and receive equipment in local station and on the subscriber

This two aspects make the radio frequency systems particularly effective in the following situations: remote subscribers or in poorly density areas and when it is necessary to rapidly install a service.

However the radio frequency spectrum is a finite resource in the sense that only a certain range of frequencies is usable for communication at any given level of technology. Although technological advances continue to expand the range of usable frequencies, the fundamental properties of radio waves and the tradition, make some radio frequencies more useful, and hence more valuable, than others. As we know the equipment and transmission characteristics in the range from a few hundreds of Megahertz to a couple of Gigahertz are especially attractive for many fixed and mobile services. There are some kind of communications that make possible, with certain modulation methods, to share the same frequency band without causing appreciable degree of interference. This method is known as SpreadSpectrum (SS) modulation and is particularly useful in conjunction with the Code Division Multiplex Access (CDMA) technique. Beyond the reuse of the same frequency band, this modulation has others attractive features: resists to intentional and non-intentional interference, can eliminate or alleviate the effect of multipath propagation and offers a certain degree of privacy, due to the use of pseudo-random spreading codes [2].

These techniques have been developed to increase military communications security due essentially to low power densities and to the code modulation making this

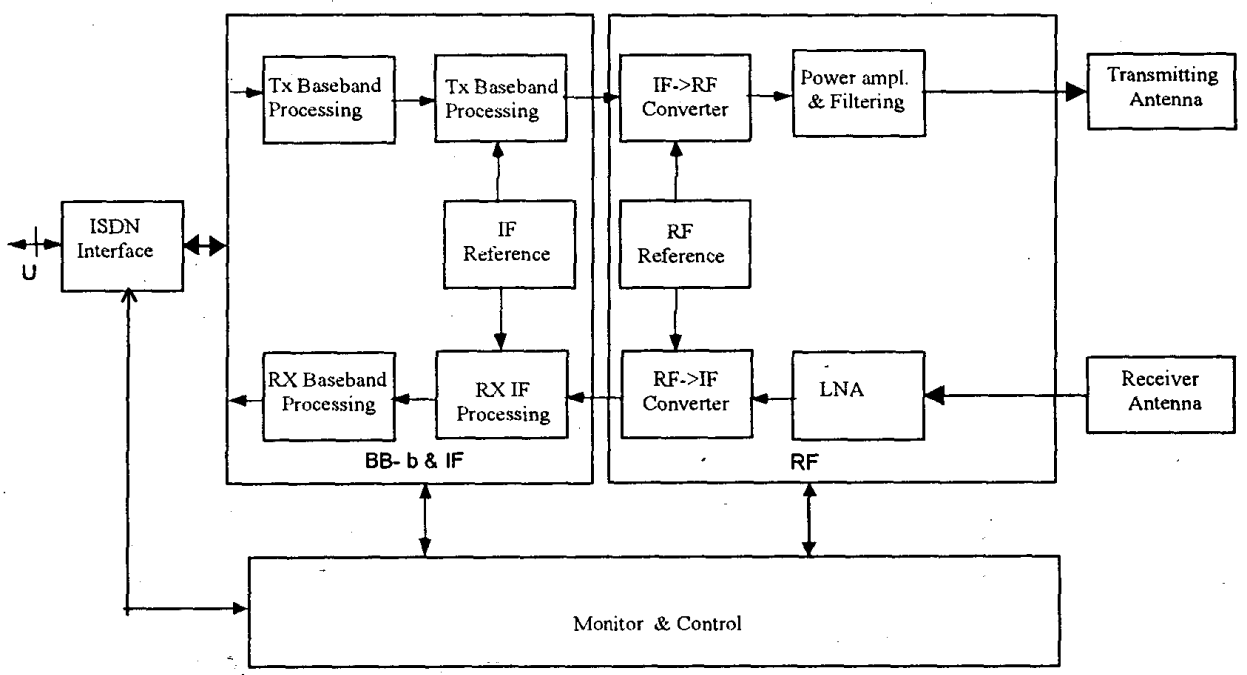

Fig. 2 Block Diagram of the transceiver 
signal noise like.

Actually the greatest interest in spread spectrum systems is in multiple access applications where a lot of users share a unified band.

The spread spectrum modulation has characteristics that make this type of modulation very attractive to be used in local loop network.

\section{System Description}

The system insertion within the ISDN reference configuration is shown in Fig. 1. The system's objective is to provide a transparent connection between two $U$ interfaces, by using the radio channel as the transmission medium. The full duplex connectivity is ensured through FDMA division between the uplink and downlink.

In a first phase a point to point connection is developed without using power control at the subscriber's premises. This will be added in a second phase while maintaining the first phase design, in order to provide a full system that accommodates a total of 10 simultaneous connections in a point to multipoint connection.

From the transmission point of view the main functional blocks of the transceiver either at the base station or subscriber's premises are shown in Fig. 2.

The functions that are provided by the various blocks are the following.

The function of the ISDN interface is to separate the two-way $2 \mathrm{~B} 1 \mathrm{Q}$ signals from the $\mathrm{U}$ interface and perform the conversion $2 \mathrm{BIQ} \rightarrow$ binary for transmission and binary $->2 \mathrm{~B} 1 \mathrm{Q}$ at the reception. Besides these two main functions, the ISDN interface also inserts (for transmission) some bits for control and monitorization and removes them in the reception.

Following the ISDN interface, the transceiver consists of one transmitter and one receiver along with a block that is designated by monitor $\&$ control.

In the transmitter we have first the binary processing, whose main functions are to perform the spreading operation. In order to accommodate in the second phase 10 simultaneous users, a code length of 64was selected. To ensure the possibility of 10 users the codes are found by extensive search and programmed bit by bit. After the spreading operation was performed, the data enters the IF processing block where the modulation and IF filtering is performed: The modulation chosen was QPSK, which using a bandwidth $30 \%$ in excess of the Nyquist bandwidth leads to a radio frequency bandwidth of about $16 \mathrm{MHz}$. The IF was fixed to be at $70 \mathrm{MHz}$, and the implementation resorts to commercially available components.

After modulation the IF signal is converted to the RF frequency $(1476 \mathrm{MHz}$ at the subscriber's premises and $1524 \mathrm{MHz}$ at the base station), amplified and filtered. The output power specified assuming no power control at the subscriber is $0.5 \mathrm{~W}$, and again the various circuits are implemented resorting to commercially available devices.

In the reception path the blocks perform the opposite functions. The first module is the LNA and is designed to provide a sensitivity of $-80 \mathrm{dBm}$ for a probability of error $P_{e}=10^{-7}$. The signal is then linearly amplified using common MMIC's. The signal after the appropriate amplification is downconverted to the IF of $70 \mathrm{MHz}$. At the IF stage the signal is kept to a constant level through the use of an AGC amplifier, which is designed to provide a dynamic range of about $30 \mathrm{~dB}$. The $\mathrm{I}$ and $\mathrm{Q}$ components are then despreaded, through correlation with the locally generated Gold code after the code sync is performed. The despreaded I and Q components are then passed through binary decision circuits and then converted in a series data stream at $192 \mathrm{Kbps}$. The probability of error that is specified is the one for cable connections, i.e. $P_{e}<10^{-7}$ [1]. Finally the data is sent to the ISDN interface where the control and monitorization bits are removed, sent to the monitor \& control block, and the signal converted into a $2 \mathrm{~B} 1 \mathrm{Q}$ format.

\section{Description of the IF and RF System} A-The transmitter

As can be seen in figure 3 the IF as a QPSK modulator driven by a synthesized $(70 \mathrm{MHz})$ oscillator, controlled by a $14 \mathrm{MHz}$ crystal oscillator. To eliminate spurious response of the QPSK modulator a band pass filter (60$80 \mathrm{MHz}$ ) is used.

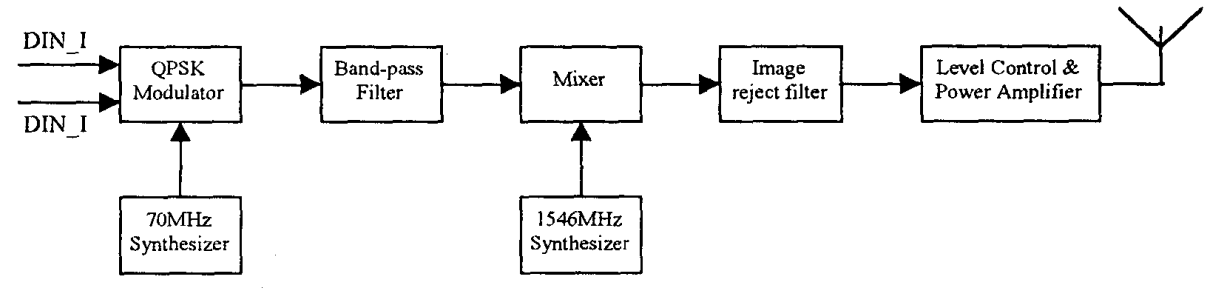

Fig. 3 Block Diagram of the Emitter 


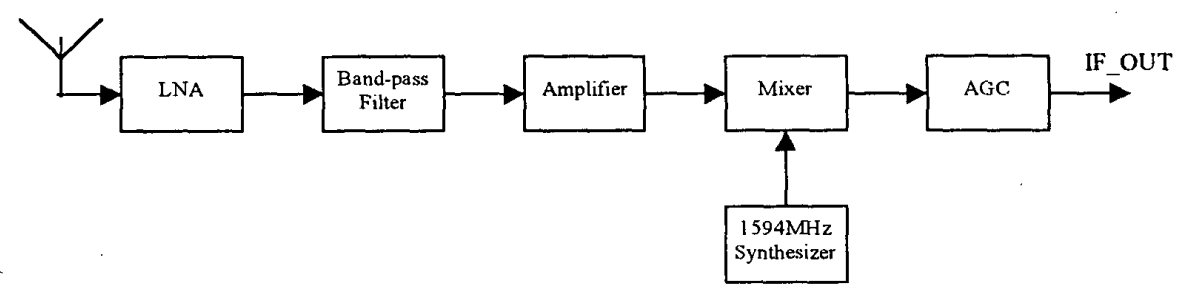

Fig. 4 Block Diagram of the Receiver

As all users employ the same frequency band the radio frequency reference is fixed. To achieve this, a fixed ratio (256) synthesizer is used. The reference crystal was adjusted for this specific purpose.

After mixing the IF signal and the RF reference the product signal is filtered by a microstrip line filter and amplified. The characteristic of this filter is critical once the IF frequency is low; a rejection of $20 \mathrm{~dB}$ is achieved at the image frequency.

In the amplifier chain a controlled gain amplifier is used to adjust the power at the local station. With this procedure the transmitted power signal is adjusted so that the power density of the received signal from each subscribers, at local station, could be of the same order of magnitude. At maximum level the emitter power is $27 \mathrm{dBm}$ obtained from a commercial MMIC circuit.

$\mathrm{B}-\mathrm{The}$ receiver

The incoming signal is first amplified in a low noise amplifier, followed by a band pass filter and another amplifier. This rejection image filter is design in microstrip technology. The overall gain of this amplifier block is $44 \mathrm{~dB}$, and some care must be taken to prevent oscillations.

After amplification the signal is mixing with a reference signal from a synthesized oscillator. This RF reference, like the one of the transmitter is based on a fixed dividing synthesizer and a specific crystal was order to obtain the pretended frequency.

Before proceeding to the demodulation an automatic gain amplifier is used to guarantee a constant level at the input of the demodulator.

\section{Conclusions}

In this communication we have considered the use of direct sequence spread spectrum for radio transmission the local loop.

We have pointed the main advantages that radio links may bring, namely in terms of ease of deployment and flexibility, which turn it advantageous for remote or scarcely populated areas, and temporary connections. This potential of radio carries us to the development of a project intended to provide a radio connection for basic ISDN services.

The main aspects and issues of the project were outlined, where it was focused the advantages that led to the choice of a spread-spectrum transmission technique, the main blocks that constitute the system were briefly described and the RF blocks were detailed.

\section{Acknowledgements}

This project is partially funded by Portugal Telecom and coordinated by its research center CET (Centro de Estudos de Telecomunicações), and the authors would like to thank CET for allowing this publication, and the colleagues H. Teixeira de Sousa and P. Mão Cheia for helpful discussions.

\section{References}

[1] CCITT Recommendation G.961, "Digital Transmission System on Metallic Local Lines for ISDN Basic Rate Access".

[2] A. Lam, S. Tantaratana, Theory and Applications of Spread-Spectrum Systems, IEEE/EAB, May 1994

[3] R. L. Pickholtz, D. L. Schilling, L. B. Milstein, "Theory of Spread-Spectrum Communications - A Tutorial", IEEE Transactions on Communication, Vol. Com-30, no. 5, pp.855-884, May. 1982.

[4] J. A. Salehi, "Emerging optical code-division multiple access communication systems", IEEE Network, pp. 3139, March 1989.

[5] V. K. Garg and E. L. Sneed, "Digital Wireless Local Loop System", IEEE Communication Magazine, pp. 112-115, Oct. 1996 